\title{
The clinical features of osteogenesis imperfecta resulting from a non-functional carboxy terminal proal(I) propeptide of type I procollagen and a severe deficiency of normal type I collagen in tissues
}

\author{
W G Cole, P E Campbell, J G Rogers, J F Bateman
}

\begin{abstract}
The features of a baby with lethal perinatal osteogenesis imperfecta (OI II), owing to a frameshift mutation that resulted in the production of a truncated and functionless carboxy terminal propeptide of the proal(I)chain of type I procollagen, were studied. The baby (0I26) was heterozygous for an insertion of a single uridine nucleotide after base pair 4088 of the preproal(I) mRNA of type I procollagen. Only normal type I collagen was incorporated into the extracellular matrix of bone and dermis resulting in a type I collagen content of about $20 \%$ of control tissues. The baby was born at 35 weeks' gestation and died shortly afterwards. He was small and had the radiographical features most like those of OI IIB. The skeleton was poorly ossified. The ribs were discontinuously beaded and the femora were broad with multiple healed fractures of the diaphyses and metaphyses. Other long bones had broad metaphyses with overmodelled diaphyses. The calvarium contained many hundreds of wormian bones. Histological examination showed grossly deficient endochondral and intramembranous ossification. The bone was of a woven type without evidence of lamellar bone or Haversian systems and the osteoblasts did not mature into osteocytes. The cortex of the femur contained Haversian canals but
\end{abstract}

Department of Paediatrics, University of Melbourne, Royal Children's Hospital, Flemington Road, Parkville, Victoria 3052, Australia.

W G Cole, J F Bateman

Department of Anatomical Pathology, Royal Children's Hospital, Melbourne, Australia.

P E Campbell

The Murdoch Institute, Royal Children's Hospital, Melbourne, Australia.

J G Rogers

Correspondence to Professor Cole.

Received for publication 20 February 1990.

Accepted for publication 27 March 1990. they were surrounded by loose collagen fibres and a mosaic pattern of woven bone and islands of cartilage. We propose that OI IIB can be subclassified into two groups, one with helical mutations and both normal and mutant type $I$ collagen in the tissues, and the other with carboxy terminal propeptide mutations and a severe type I collagen deficiency, but without mutant collagen in the tissues.

Osteogenesis imperfecta (OI) is a heterogeneous genetic disorder of the connective tissues. ${ }^{1}$ The major phenotypic features include bone fragility, abnormal blueness of the sclerae, dentinogenesis imperfecta, and premature deafness. Patients with OI have been classified into four major groups according to their clinical, radiographical, and inheritance patterns. ${ }^{1}$ The most severe form, lethal perinatal type II osteogenesis imperfecta (OI II), is the focus of this report.

Biochemical abnormalities of type I procollagen have been found in many patients with $O I$ and particularly in those with OI II. ${ }^{2}$ The mutations involve the COLlAl gene that encodes the proal(I) chains and the COL1A2 gene that encodes the proa2(I) chain of type I procollagen. In OI II, the reported mutations are heterozygous and result in insertions, deletions, or amino acid substitutions in the triple helical domain of the proal(I) chain and less frequently in the same domain of the proo $2(\mathrm{I})$ chain. ${ }^{3-8}$

Cultured dermal fibroblasts from babies with OI II produce and secrete abnormal type I procollagen, containing one or more mutant chains, and normal type I procollagen. ${ }^{9}$ Characteristically, the mutant molecules contain abnormally high levels of hydroxylated lysine residues and are poorly secreted and easily degraded, but some of them are incorporated, with normal molecules, into the extracellular matrix of tissues. ${ }^{10}$

In contrast to other reported cases of OI II, this 
report concerns a baby (OI26) with a COL1A1 mutation that did not involve the main triple helical domain of the proal(I) chain nor lead to the incorporation of a mixture of normal and mutant type I collagen in the tissues. ${ }^{1011}$

A codon frameshift mutation caused by a single uridine insertion after base pair 4088 of the preproal(I) mRNA of type I procollagen was identified in case OI26. ${ }^{11}$ The heterozygous mutation was probably the result of a thymine insertion in exon 49 of COL1A1. The frameshift resulted in a truncated prool(I) carboxy terminal propeptide in which the amino acid sequence was abnormal from valine ${ }^{1146}$ to the carboxyterminus. The propeptide lacked asparagine $^{1187}$, which normally carries an $N$ linked oligosaccharide unit, and was more basic than the normal propeptide. The distribution of cysteines was altered and the mutant propeptide was unable to form normal inter-chain disulphide bonds. Some of the mutant proal(I) chains were incorporated into type I procollagen molecules but resulted in overhydroxylation of lysine residues, increased degradation, and poor secretion, as reported in other cases of OI II. However, in case OI26 the mutant molecules were so abnormal that only normal type I collagen was incorporated into the extracellular matrix of bone and dermis, resulting in a type I collagen content of about $20 \%$ of that of control tissues. ${ }^{10}$ In this paper we describe the clinical, radiographical, and pathological features of this baby with OI II.

\section{Case report}

\section{CLINICAL HISTORY}

The proband was the second child of an unrelated 25 year old father and 23 year old mother. Both parents were healthy except that the mother had conductive deafness that did not appear to be caused by OI. This proposal was supported by the observation that collagen metabolism was normal in cultured dermal fibroblasts from both parents. ${ }^{11}$ The proband's sister was clinically and radiographically normal. There were three further pregnancies after the birth of the proband. Two resulted in the birth of normal children while one terminated spontaneously at 10 to 12 weeks' gestation. The aborted fetus appeared to have developed normally and to have a normal skeleton. The cause of the miscarriage was not determined. A paternal cousin was abnormally large at birth and had radiographical features similar to those of asphyxiating thoracic dystrophy. His development was delayed and he died at 20 months.

The pregnancy resulting in the birth of the proband was uneventful and fetal movements were considered by the mother to be normal. He was born by normal vaginal delivery at 35 weeks' gestation after spontaneous onset of labour. The baby made a few gasping respiratory movements but died 20 minutes later.

The birth weight of $1400 \mathrm{~g}$, crown-heel length of

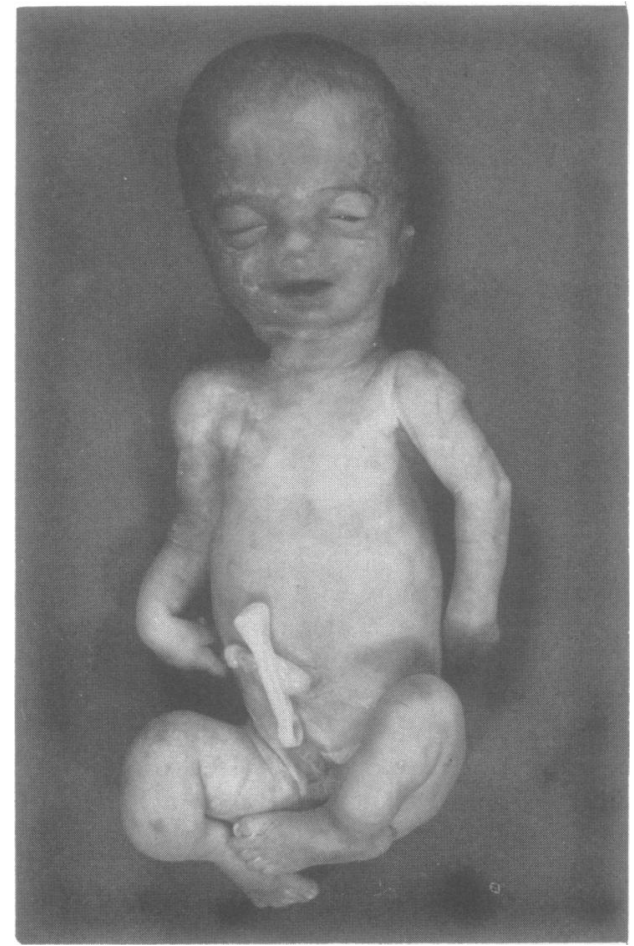

Figure 1 OI26: clinical appearance.

$40.5 \mathrm{~cm}$, and head circumference of $27 \mathrm{~cm}$ were below the 10th centile for this length of gestation. ${ }^{12}$ The baby had a relatively large calvarium, widely spaced eyes, and a broad nasal bridge (fig 1). The calvarium was extremely soft with no palpable bone except in the frontal and occipital regions. He had a small, triangular face and pinched nose as well as a small mouth and mandible. The chest was narrow proximally and flared distally. The spinal shape was normal. The upper limbs were slender and showed minimal deformity, although there was crepitus from fractures. The arms measured $13 \mathrm{~cm}$ from the acromion to the tips of the fingers. The thighs and shins were bowed and there was crepitus from multiple fractures of the femora and tibiae. The legs measured $13 \mathrm{~cm}$ from the greater trochanter to the heel. The hands and feet were of normal shape.

\section{RADIOGRAPHICAL FEATURES}

The skeleton was poorly ossified. All long bones were smaller than normal and showed broad metaphyses, abnormal modelling, and thin cortices. There were widespread fractures in varying stages of healing and these features were most obvious in the lower limbs and ribs. 


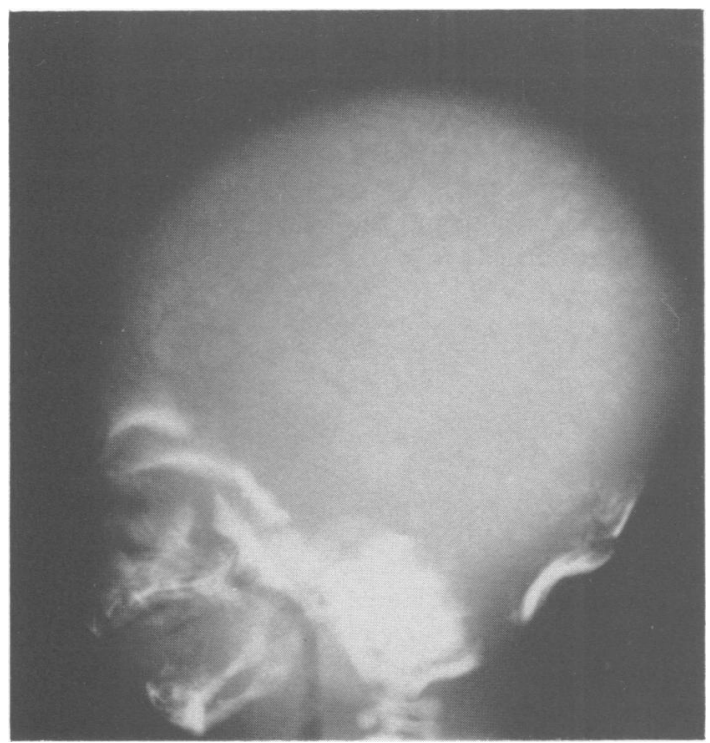

Figure 2 Lateral radiograph of the skull showing many hundreds of wormian bones. The base of the skull, orbits, face, and jaw are better ossified than the calvarium.

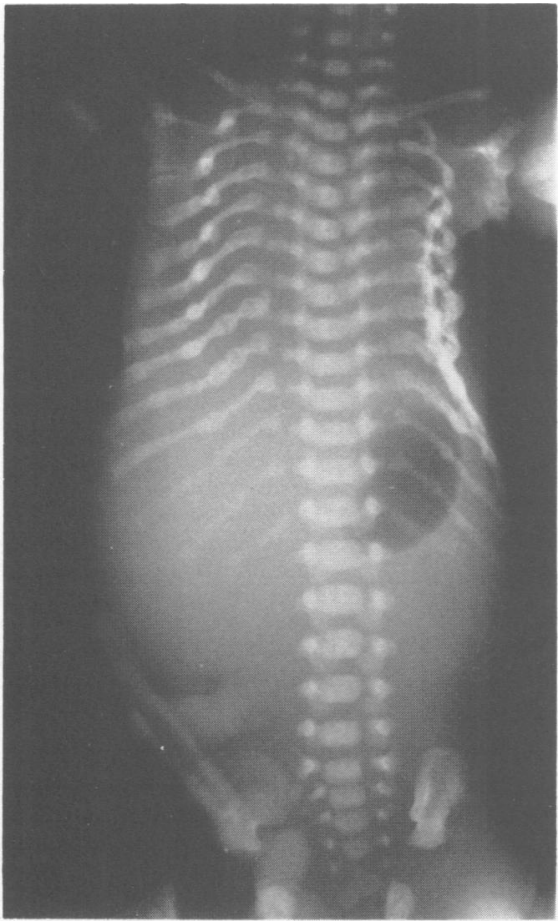

Figure 3 Anteroposterior radiograph of the torso. The ribs are discontinuously beaded with distortion of the chest wall. The vertebrae and pelvis are well formed.
The calvarium consisted of many hundreds of wormian bones, but the occiput, base of skull, and orbits were better ossified (fig 2). The maxilla and mandible were hypoplastic and poorly ossified.

The vertebrae were well formed with minimal, if any, platyspondyly (fig 3). The ribs were discontinuously beaded with healing fractures, and the upper ribs were invaginated while the lower ones were flared. Each scapula was of normal shape and showed a transverse fracture of the mid-axillary border.

The bones of the upper limbs had mildly broadened metaphyses with some metaphyseal fractures and almost normal modelling of the diaphyses (fig 4). The radius, shown in fig 4 , was poorly modelled as the diaphysis was widened owing to several malunited fractures.

The pelvic shape was normal (fig 5). The femora showed broad metaphyses with healing metaphyseal fractures. The shafts varied in their diameters but were generally broad and distorted from malunited fractures and subperiosteal new bone formation. The tibial metaphyses were also broad and fractured at their narrowest points. The fibulae were also abnormal.

\section{PATHOLOGICAL FEATURES}

Necropsy showed that the viscera were normally formed and the testes were in the posterior abdominal wall. The skin was soft and thin. The calvarium

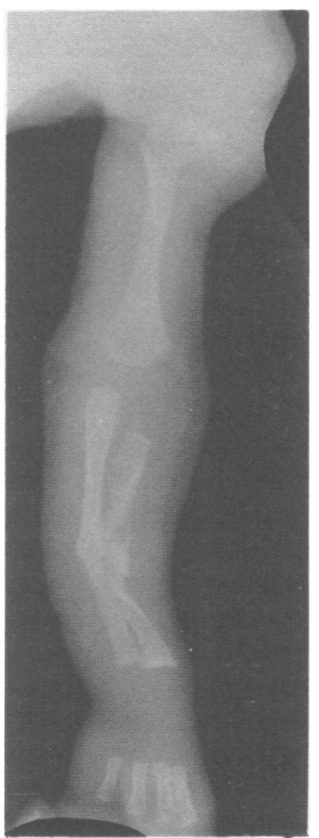

Figure 4 The humerus, ulna, and metacarpals are well formed. The proximal humeral and distal ulnar metaphyses are mildly broadened and are fractured. The radius is broad and deformed owing to multiple fractures. 
consisted of a membrane containing tiny flakes of calcified tissue corresponding to the wormian bones. This membrane consisted of thickened dura that was fused with the galea aponeurotica. The base of the skull was better formed and ossified. The ribs showed

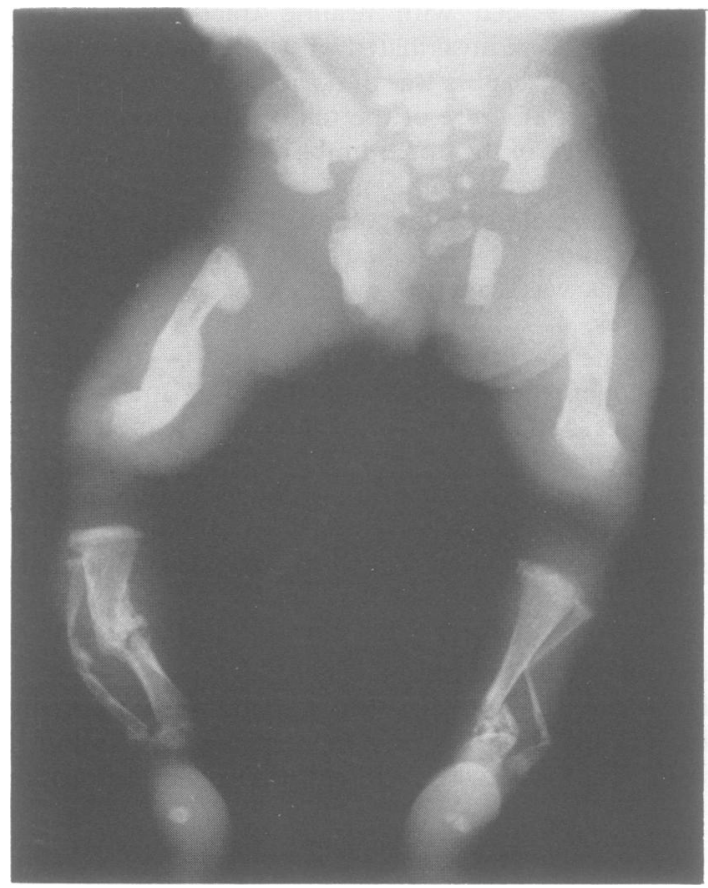

Figure 5 Radiograph of the pelvis and legs. The femora are broad with multiple healing fractures. The tibial and fibular metaphyses are broad and fractured. Their shafts are overmodelled and fractured. multiple healing fractures which produced lumps on their pleural surfaces. The vertebrae were of normal shape and size, while the femora were broad and contained healing fractures.

The placenta weighed $230 \mathrm{~g}$ and had a diameter of $10 \mathrm{~cm}$ and thickness of $2 \mathrm{~cm}$. The cut surface was soft and homogeneous and the fetal and maternal vessels appeared normal.

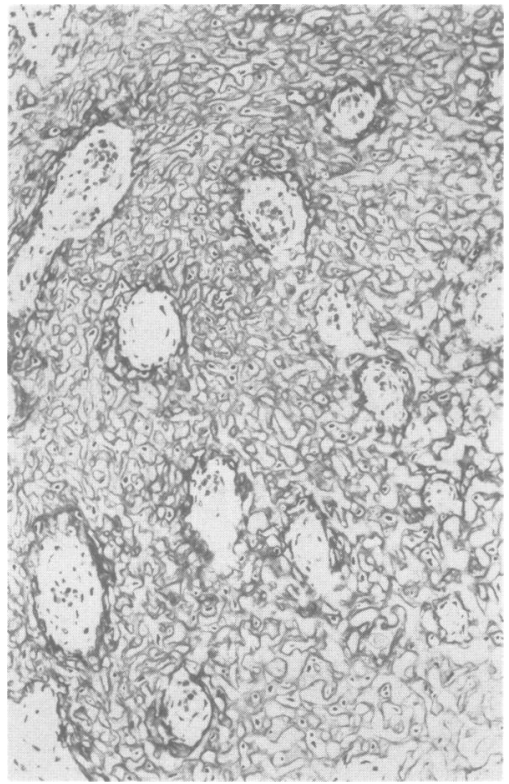

Figure 7 Section of femoral cortex from OI26. The cortical bone shows thin seams of woven bone surrounding entombed islands of cartilage giving a mosaic appearance. Poorly formed Haversian canals are present. (Haematoxylin and eosin.)
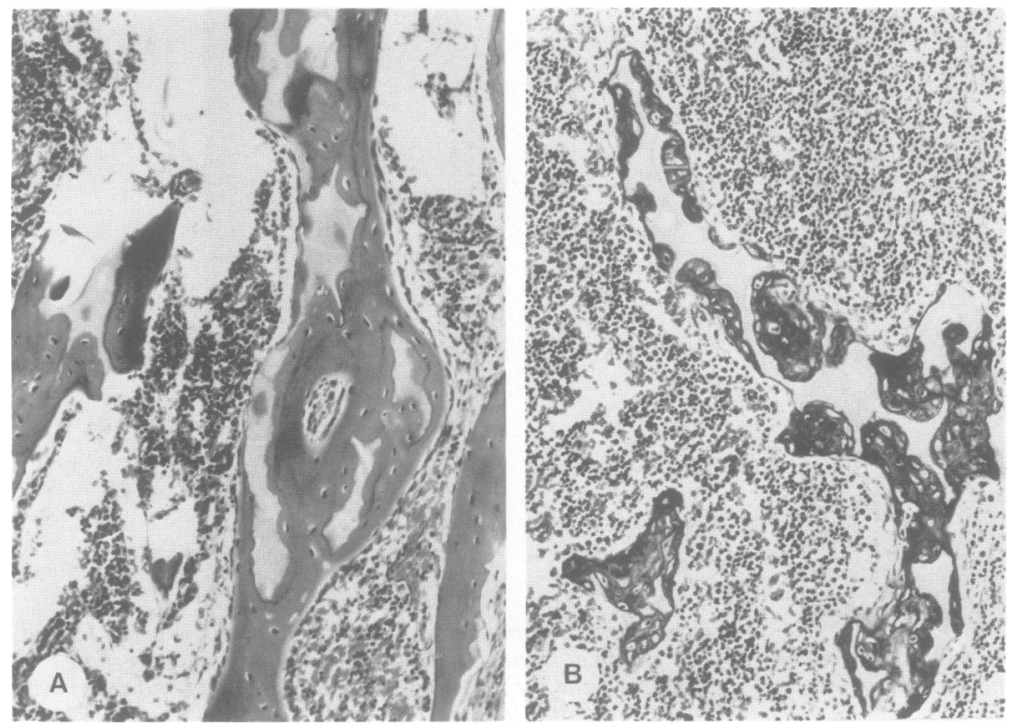

Figure 6 Trabecula of the ribs. (A) Control rib matched for age, birth weight, and site (haematoxylin and eosin). The trabecula are thick and close together. Most of the cartilage has been replaced with lamellar bone. The surfaces of most trabecula are covered with a continuous layer of fat osteoblasts and a thick layer of osteoid. The cells within the lamellar bone have a more mature osteocytic appearance. (B) OI26 rib (haematoxylin and eosin). The trabecula are thin and consist of a central cartilage core with an incomplete surface layer of very thin woven osteoid and pockets of woven bone. There is an overabundance of marrow. 
Histological examination of a costochondral junction showed that the cartilaginous part of the growth plate was normal. However, ossification was grossly deficient. The osteoid seams along the cartilaginous trabecula were extremely thin (fig 6). Cartilage cores persisted in trabecula found throughout the full length of the diaphysis. All the bone was of a woven type without evidence of lamellar bone or Haversian systems. In addition, the osteoblasts maintained their plump and immature features without evidence of maturation to osteocytes. Healing

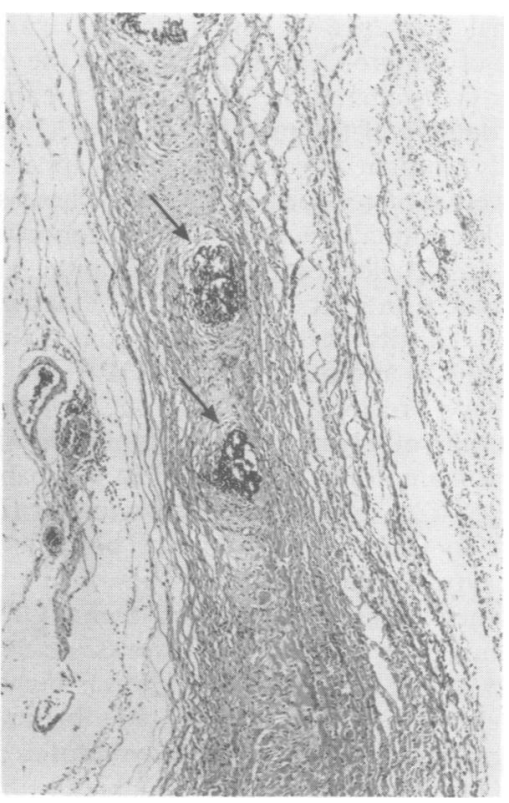

Figure 8 Calvarium of OI26 showing several centres of ossification in a fused membrane. The intramembranous centres of ossification consist of loose woven bone. fractures consisted of a proliferation of cartilage and irregular fibrosis but with minimal ossification.

The histological appearance of the femur was similar to that of the rib. However, the cortex consisted of woven bone surrounding entombed islands of cartilage giving an unusual mosaic appearance similar to that seen in osteopetrosis (fig 7). Poorly formed Haversian canals were present with a central blood vessel and a mantle of very pale staining, loose collagen fibres. As in the rib, fractures contained excessive amounts of cartilage and fibrous tissue and little osteoid. The vertebral bodies also showed similar appearances to the femur and rib.

Sections through the calvarium showed that it consisted of a membrane, presumably fused galea aponeurotica and dura mater, containing small, isolated islands of ossification (fig 8). The bone was of a woven type but the ossification centres did not contain cartilage.

The dermis from case OI26 was about half the thickness of dermis from a matched control sample (fig 9). The papillary and reticular layers of the dermis of OI26 were indistinguishable from each other. The collagen fibres were slender and the fibroblasts were numerous but appeared normal. The amount of elastin appeared to be reduced.

The connective tissue of the pancreas, skeletal muscle, and epicardium stained poorly. The collagen fibres were fine and widely spaced. Sections of lung showed very thin and delicate alveolar septa, even though the lung tissue was atelectatic, suggesting that the amount of collagen was reduced.

The eye and aorta were also studied. The globe was of normal size. The scleral thickness varied from 200 to $400 \mu$ and its collagen was loosely woven. The internal structure of the eye was normal although the lens detached very easily during processing, suggesting possible fragility of its supporting tissues. The media
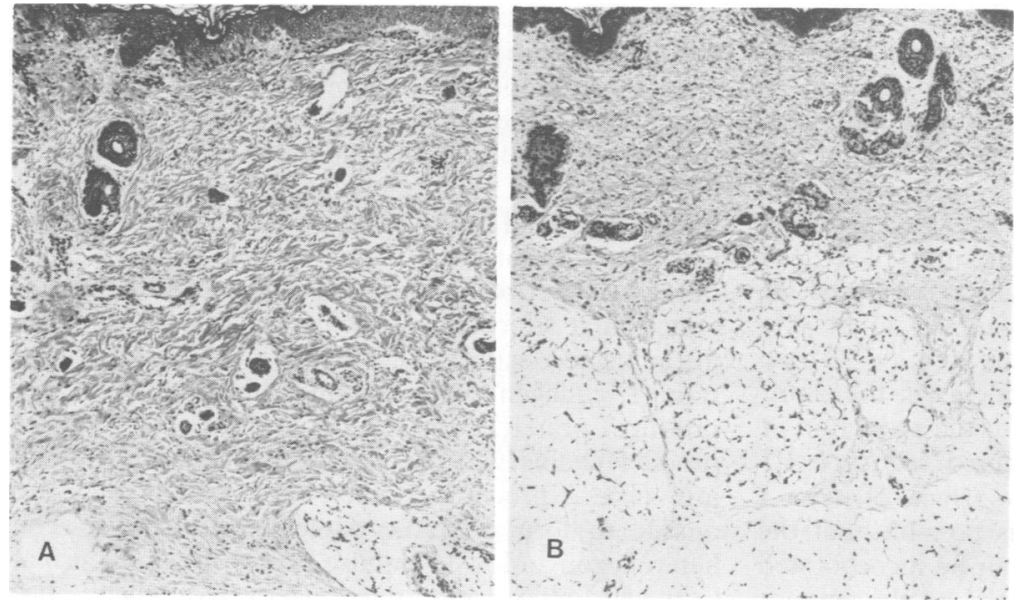

Figure 9 The skin from $(A)$ control and $(B) O I 26$. The epidermis and dermis of OI26 are thinner than age and site matched skin. The collagen fibres in OI26 are also finer than the control fibres. 
of the thoracic aorta had an average thickness of $400 \mu$. The elastic fibres were normally formed. Collagen, as shown by Masson trichrome staining, was sparse.

\section{Discussion}

Type II or perinatal lethal osteogenesis imperfecta has been subclassified into types IIA, IIB, and IIC. ${ }^{13} 14$ Type IIA has broad, crumpled long bones and ribs with severe platyspondyly, very poor ossification of the skull, and perinatal death. Type IIB has discontinuously beaded ribs, crumpled femora, angulated tibiae, better modelled humeri, more normal vertebrae, better ossification of the skull, and survival may occur beyond the perinatal period. Type IIC has thin, cylindrical, and dysplastic long bones and thin, beaded ribs with stillbirth. OI 26 had clinical and radiographical features that were most like those of type IIB.

The main phenotypic features of case OI26 involved the bone and dermis. Previous studies showed that the bone matrix only contained normal type I collagen, but the amount was reduced to about $20 \%$ of normal. ${ }^{10}$ This deficiency accounts for the gross fragility of the bones and for some of the morphological changes. For example, the reduced amount of collagenous matrix accounts for the smallness of the bones and the grossly deficient endochondral and intramembranous ossification. It also accounts for the overmodelling of many of the long bones as this process normally involves the removal of metaphyseal bone and the formation of new diaphyseal bone. New bone formation, with widening of the diaphyses, was stimulated by intrauterine fractures but the ossified matrix was still grossly deficient.

Case OI26 also lacked lamellar bone and Haversian systems and the osteoblasts did not mature into osteocytes. Pseudo-Haversian systems, consisting of a central vascular canal and surrounding collagen fibres and woven bone, were observed in the femoral cortex. The failure of the bone of case OI26 to form a well organised matrix suggests that a critical amount of normal type I collagen in the matrix is required for these organisational and maturational events to occur.

Previous studies also showed that the skin contained only about $14 \%$ of the normal amount of type I collagen and $21 \%$ of the normal amount of type III collagen, although there did not appear to be any primary abnormality in type III collagen. ${ }^{10} 11$ These deficiencies account for the thinness of the skin. However, the mechanism leading to the reduction of type III collagen was not determined. As type III collagen is found on the outer surface of type I collagen fibres in the dermis, it is possible that the amount of type III collagen that can be incorporated into the composite fibres is regulated by the amount of available type I collagen. ${ }^{15}$ In contrast, the bone of OI26 contained a small percentage of type III collagen which was not detectable in normal bone matrix. ${ }^{10} \mathrm{It}$ is likely that the type III collagen was located in the abundant marrow and soft tissue rather than in the calcified matrix of OI26 bone. ${ }^{10}$ The differences in the proportions of type III collagen in the dermis and bone suggest that the observed type III collagen anomalies were secondary events in the pathogenesis of the disease.

The molecular defect and its consequences in OI26 were different from those reported in other cases of OI II. Firstly, the mutation in OI26 resulted in an abnormal sequence of the carboxy terminal propeptide of the proal(I) chain, whereas the mutations in other cases altered the sequence of the main triple helical domain of the proal(I) or prow2(I) chains. ${ }^{3-8}$ Secondly, mutant molecules in OI26 appeared to be exclusively targeted for degradation, whereas in cases with helical mutations a significant proportion of the mutant molecules escaped degradation and were incorporated into the extracellular matrix of the tissues. ${ }^{10}$ Thirdly, there were some clinical differences between OI26 and other cases of OI II.

We have previously characterised a heterozygous substitution of glycine ${ }^{976}$ by arginine in the triple helical domain of the proal(I) chain in a baby $(\mathrm{OI} 30)$ who, like OI26, was classified as having OI IIB. ${ }^{6}{ }^{16}$ In contrast to OI26, this baby had both normal andō mutant type I collagen in the tissues. There were also? some other differences between them. OI30 was a short limbed dwarf who at $\mathbf{4 0}$ weeks' gestation only weighed $1091 \mathrm{~g}$. In contrast, OI26 was bigger for his gestational age and his skeleton was better formed. The significance of these apparent differences will need to await further studies of babies with OI IIB, but at this stage we propose that OI IIB may be subclassified into those babies with helical mutations and normal and mutant type I collagen in the tissues and those with carboxy terminal propeptide mutations and a severe type I collagen deficiency, but without mutant collagen in the tissues.

The results of this and other studies provide an insight into the phenotypic expression of type I collagen deficiencies. The most extreme deficiency is illustrated by the homozygous Mov-13/Mov-13 mouse, in which both COL1Al alleles are inactivated by retroviral insertion into intron $1 .^{1718}$ They developed normally until the 12th to 14 th day of gestation at which time vascular ruptures resulted in intrauterine death. ${ }^{17}$ The tissues of these mice lack type I collagen and cultured fibroblasts do not produce prool(I) mRNA or chains. The excess proa2(I) chains, which would normally be incorporated into type I procollagen, are destroyed as they are unable to form a stable triple helix at $37^{\circ} \mathrm{C}$. The heterozygous Mov-13 mouse, carrying one normal COLlAl allele and one inactivated allele, has 
morphological anomalies, decreased bone strength, premature deafness, and about half normal amounts of type I collagen in the tissues. ${ }^{19}$ These features are very similar to those of human type I OI (OI I), the classical autosomal dominant mild type of this disease. ${ }^{20}$ Cultured fibroblasts from many cases of human OI I have decreased levels of COL1Al mRNA and reduced production of proal(I) chains. ${ }^{20}$ The molecular defects causing the apparent lack of expression of one COLlAl allele in these cases are largely unknown. However, in one case there was a frameshift mutation in the $3^{\prime}$ region of the COLlAl gene, but unlike OI26, the mutant prool(I) chain that could be translated in vitro could not be detected in the cell and did not associate with normal chains. ${ }^{20}$ These reports and our findings in OI26 suggest that reduction in the amount of normal type I collagen in the tissues from $50 \%$ to about $20 \%$ means the difference between developing mild OI I and lethal OI II.

Type I collagen deficiency in the tissues can also arise from mutations involving the COL1A2 gene. A homozygous frameshift mutation owing to a four base pair deletion in the COL1A2 gene was reported in a child with a severe but viable type III form of OI. ${ }^{21}$ This mutation resulted in an abnormal proa2(I) carboxy terminal propeptide in which the sequence of the last 33 amino acids was incorrect. The mutant proa2(I) chains were unable to associate with the normal prool(I) chains and were degraded, but the remaining normal pro $\alpha \mathrm{l}(\mathrm{I})$ chains could form trimeric $\alpha \mathrm{l}(\mathrm{I})$ molecules. These trimers were able to compensate, to some extent, for the total deficiency of type I collagen in this child. The heterozygous parents had radiographical evidence of osteoporosis. ${ }^{22}$ In contrast, the heterozygous mutation in OI26 resulted in the loss of about half of the normal proal(I) chains, and the excess normal proa2(I) chains were not able to form proo2(I) trimers as such molecules are unstable at body temperature.

Studies of further babies with OI and transgenic mice with reduced amounts of normal type I collagen in the tissues should provide additional insights into the phenotypic expression of varying severities of type I collagen deficiency.

This work was undertaken with grants from the National Health and Medical Council of Australia, the Royal Children's Hospital Research Foundation, and the Osteogenesis Imperfecta Foundation.

1 Sillence DO, Senn AS, Danks DM. Genetic heterogeneity in osteogenesis imperfecta. 7 Med Genet 1979;16:101-16.

2 Byers PH, Tsipouras P, Bonadio JF, Starman BJ, Schwartz RC. Perinatal lethal osteogenesis imperfecta (OI type II): a bio- chemically heterogeneous disorder usually due to new mutations in the genes for type I collagen. Am F Hum Genet 1988;42: 237-48.

3 Chu ML, Williams CJ, Pepe G, Hirsch JL, Prockop DJ, Ramirez F. Internal deletion in a collagen gene in a peripheral lethal form of osteogenesis imperfecta. Nature 1983;304:78-80

4 Bateman JF, Chan D, Walker ID, Rogers JG, Cole WG. Letha perinatal osteogenesis imperfecta due to the substitution of arginine for glycine at residue 391 of the $\alpha \mathbf{l}(\mathrm{I})$ chain of type I collagen. F Biol Chem 1987;262:7021-7.

5 Bateman JF, Lamande SR, Dahl HHM, Chan D, Cole WG Substitution of arginine for glycine 664 in the collagen $\alpha 1(I)$ chain in lethal perinatal osteogenesis imperfecta. 7 Biol Chem 1988;263:11627-30.

6 Lamande SR, Dahl HHM, Cole WG, Bateman JF. Characterization of point mutations in the collagen COL1Al and COL1A2 genes causing lethal perinatal osteogenesis imperfecta. F Biol Chem 1989;264:1589-12.

7 Cohn DH, Byers PH, Steinmann B, Gelinas RE. Lethal osteogenesis imperfecta resulting from a single nucleotide change in one human pro alpha $\mathbf{1}(\mathrm{I})$ collagen allele. Proc Natl Acad Sci USA 1986;83:6045-7.

8 Constantinou CD, Nielsen KB, Prockop DJ. A lethal variant of osteogenesis imperfecta has a single base mutation that substitutes cysteine for glycine 904 of the alpha l(I) chain of type procollagen. The asymptomatic mother has an unidentified mutation producing an overmodified and unstable type I procollagen. F Clin Invest 1989;83:574-84.

9 Bateman JF, Mascara T, Chan D, Cole WG. Abnormal type I collagen metabolism by cultured fibroblasts in lethal perinatal osteogenesis imperfecta. Biochem f 1984;217:103-15.

10 Bateman JF, Chan D, Mascara T, Rogers JG, Cole WG. Collagen defects in lethal perinatal osteogenesis imperfecta. Biochem $\mathcal{f}$ 1986;240:699-708.

11 Bateman JF, Lamande SR, Dahl HHM, Chan D, Mascara T, Cole WG. A frameshift mutation results in a truncated nonfunctional carboxy-terminal prod l(I) propeptide of type I collagen in osteogenesis imperfecta. $\mathcal{F}$ Biol Chem 1989;264: $10960-4$.

12 Kitchen WH, Robinson HP, Dickinson AJ. Revised intrauterine growth curves for an Australian hospital population. Aust Paediatr f 1983;19:157-61.

13 Sillence DO, Barlow KK, Garber AP, Hall JG, Rimoin DL. Osteogenesis imperfecta type II. Delineation of the phenotype with reference to genetic heterogeneity. Am $\mathcal{J}$ Med Genet 1984;17:407-23.

14 Thompson EM, Young ID, Hall CM, Pembrey ME. Recurrence risks and prognosis in severe sporadic osteogenesis imperfecta. f Med Genet 1986;24:390-405.

15 Keene DR, Sakai LY, Bachinger HP, Burgeson RE. Type III collagen can be present on banded collagen fibrils regardless of fibril diameter. F Cell Biol 1987;105:2393-402.

16 Cole WG, Chow CW, Rogers JG, Bateman JF. The clinical features of three babies with osteogenesis imperfecta resulting from the substitution of glycine by arginine in the proal(I) chain of type I procollagen. F Med Genet 1990;27:228-35.

17 Lohler J, Timpl R, Jaenisch R. Embryonic lethal mutations in mouse collagen 1 gene causes rupture of blood vessels and is associated with erythropoietic and mesenchymal cell death. Cell 1984;38:597-607.

18 Schnieke A, Dziadek M, Bateman J, et al. Introduction of the human pro alpha $\mathrm{I}(\mathrm{I})$ collagen gene into pro alpha $\mathrm{I}(\mathrm{I})$ deficient Mov-13 mouse cells leads to formation of functional mousehuman hybrid type I collagen. Proc Natl Acad Sci USA 1987;84:764-8.

19 Bonadio J, Saunders TL, Tsai E, et al. Transgenic mouse model of the mild dominant form of osteogenesis imperfecta (submitted).

20 Willing MC, Cohn DH, Byers PH. Frameshift mutation near the $3^{\prime}$ end of the COLIAl gene of type I collagen predicts an elongated proal(I) chain and results in osteogenesis imperfecta type I. F Clin Invest 1990;85:282-90.

21 Pihlajaniemi $\overline{\mathrm{T}}$, Dickson LA, Pope $\overline{\mathrm{FM}}$, et al. Ōsteogenesis imperfecta: cloning of a pro- $\alpha 2$ (I) collagen gene with a frameshift mutation. I Biol Chem 1984;259:12941-4.

22 Nicholls AC, Osse G, Schloon HG, et al. The clinical features of homozygous $\alpha 2(\mathrm{I})$ collagen deficient osteogenesis imperfecta. f Med Genet 1984;21:257-62. 